\title{
Vascular dementia: a diagnosis running out of time ${ }^{\dagger}$
}

\author{
ROBERT STEWART
}

\author{
Background The concept of vascular \\ dementia has a long history but its \\ usefulness as a diagnostic category has \\ been called into question.
}

\begin{abstract}
Aims To evaluate vascular disease as a risk factor for dementia and the interface between cerebrovascular pathology and Alzheimer's disease.
\end{abstract}

Method The literature on this topic was selectively reviewed and synthesised.

\section{Results Risk factors for \\ cerebrovascular disease are also risk factors for dementia. However, the course of dementia, once it has developed, appears to be frequently determined by Alzheimer's disease.}

\section{Conclusions As a public health} measure, modification of vascular risk represents a potentially powerful means to prevent dementia through delaying its onset. However, an effect on progression of dementia, once it has developed, has yet to be established. The traditional view of vascular dementia and Alzheimer's disease as distinguishable conditions is becoming steadily less tenable.

\section{Declaration of interest R.S. is} supported by a ResearchTraining Fellowship in Clinical Epidemiology from the WellcomeTrust.
Once upon a time, around the first half of the last century, late-onset dementia was viewed as an essentially homogeneous condition. The main underlying cause was assumed to be atherosclerosis: a pathological substrate for 'old age'. Pioneering work around the 1960s and early 1970s challenged this assumption, establishing the importance of Alzheimer pathological changes in the majority of cases. However, multiple cerebral infarctions also appeared to underlie a substantial proportion. This led to the division of dementia into the two conditions of Alzheimer's disease and multi-infarct dementia. This system has persisted to this day but is coming under increasing criticism, particularly from those who wish to investigate vascular disease as a risk factor for dementia. In the following sections the current status of and future directions for 'vascular dementia' are considered, both as a diagnostic category and as a heading for a broad field of research.

\section{AN OUTDATED DIAGNOSTIC SYSTEM?}

Research diagnostic criteria treat Alzheimer's disease as a diagnosis of exclusion (McKhann et al, 1984). This system may be useful for examining issues relating to this disorder as a 'pure' entity. However, it may also miss potentially important influences from factors such as vascular disease, which have been excluded by definition. In addition, having begun by excluding major vascular disease such as clinical stroke or multiple cerebral infarctions on computed tomographic imaging, studies that use this diagnostic system now have to consider what to do with evidence of more subtle, subclinical disease, such as white-matter abnormalities seen on magnetic resonance imaging. Increasingly sophisticated measurements of cerebrovascular disease in vivo raise the question of where exclusion criteria should stop. If detectable levels of vascular disease continue to be excluded, the 'pure' Alzheimer's disease group will become progressively smaller, defining an increasingly select group of persons who, for whatever reason, have remained free of any vascular disease into old age. The question, of course, is what level of vascular disease may cause dementia; this cannot be answered by using criteria that assume that it is or is not a cause.

A major problem with the concept of multi-infarct dementia has been that diagnostic criteria tried to do the same thing as those for Alzheimer's disease: that is, to define a 'pure' entity. However, while Alzheimer's disease at least has a reasonably well-defined pathological basis, the same is not true for dementia related to cerebrovascular disease. 'Vascular dementia' subsumed multi-infarct dementia as a term because of recognition of this heterogeneity, and large numbers of other subtypes have subsequently been proposed (Konno et al, 1997). These range from syndromes where dementia appears to have resulted from small, discrete 'strategic' infarctions (such as bilateral thalamic infarction or lesions of the angular gyrus), to dementia associated with more diffuse cerebrovascular disease: ischaemic or haemorrhagic, cortical or subcortical (Brun, 2000). More diffuse disease obviously exists across a spectrum of severity and with advancing age is increasingly likely to be comorbid with Alzheimer's disease, raising questions as to how (or whether) the two should be distinguished in a diagnostic system. An additional obstacle for research is that the term 'vascular dementia' makes assumptions about causation and therefore is of limited use when it comes to investigating potential causes. It would not be considered particularly noteworthy if a study was to report that people who have a stroke are more likely to have hypertension or diabetes. Similarly, it would not be very surprising if they were found to have higher mortality or higher levels of depression. The problem with 'vascular dementia' as a term is that comparisons with Alzheimer's disease or control groups are inevitably comparisons of populations with different rates of cerebrovascular disease, and it is impossible to conclude what is related to cerebrovascular disease and what is related to a particular dementia syndrome.

It is perhaps preferable to consider vascular dementia as a series of questions rather than a diagnosis. Does vascular 
disease cause dementia? What type (or types) of dementia does it cause? What can we do about it?

\section{DOES VASCULAR DISEASE CAUSE DEMENTIA?}

Systems of dementia classification have been a major obstacle for investigating this question, as previously discussed. Another problem is that age groups in which dementia is most common contain, to an extent, healthy survivors with respect to vascular disease. Also, people with both conditions together have a higher mortality and are therefore less likely to be identified in both cross-sectional and prospective studies.

Particularly high rates of dementia are observed following clinical stroke, with an approximately nine-fold increase in risk over the first year followed by a smaller, two-fold raised annual risk which has been found to persist at least 25 years later (Kokmen et al, 1996). Vascular risk factors such as hypertension, diabetes and hypercholesterolaemia have also been established as risk factors for dementia in large prospective studies (Stewart, 1998). Early reports that smoking might be a protective factor, at least for Alzheimer's disease, have not been confirmed by more rigorously designed investigations which, if anything, report a raised risk of both subtypes of dementia in smokers (Ott et al, 1998). An important issue arising from these studies concerns the stage of cognitive decline at which these factors have an effect. Raised blood pressure in mid-life is, for instance, a strong risk factor for later cognitive impairment (Elias et al, 1993). However, blood pressure in later life tends to be lower in association with dementia (Skoog et al, 1996). Therefore hypertension appears to exert its effect at an early stage in cognitive decline and the association may no longer be apparent by the time cognitive function has deteriorated sufficiently to be classified as dementia. The same pattern has been described for raised cholesterol concentrations (Notkola et al, 1998), and has important potential implications for preventive or therapeutic intervention.

\section{WHAT TYPE OF DEMENTIA DOES IT CAUSE?}

Again, traditional diagnostic criteria have hampered investigation of this question.
An increasing body of epidemiological evidence suggests that vascular risk factors such as hypertension, diabetes and hyperlipidaemia are risk factors not only for the development of vascular dementia but also for Alzheimer's disease (Stewart, 1998). Community-based research can be criticised in that neuroimaging has not been routinely used in diagnostic assessment, so that subclinical levels of cerebrovascular disease may have been missed and 'mixed dementia' misidentified as Alzheimer's disease. This issue, however, should be more a consideration of mechanisms by which vascular risk factors are associated with dementia rather than one of arbitrarily defined diagnostic categories. Post-stroke studies have found that dementia appears to follow an Alzheimer's disease-like course in the majority of cases (Kokmen et al, 1996) and that approximately $10 \%$ of people with post-stroke dementia have had gradually deteriorating cognitive function before the stroke occurred (Pohjasvaara et al, 1999). Since Alzheimer's disease has a long latency period, it can be assumed that a substantial further proportion may have had subclinical disease.

What is becoming increasingly apparent is that dementia associated with vascular disease frequently resembles Alzheimer's disease in its clinical course and, in many cases, is not obviously explained by a multiple infarction mechanism. One possibility is that vascular processes cause a gradually progressive dementia syndrome. However, recent pathological studies have suggested that isolated cerebrovascular disease (i.e. with no Alzheimer lesions) is rarely found in association with dementia (Hulette et al, 1997). These findings contrast with those from the early post-mortem series, although it should be borne in mind that people with dementia in the late 1960s would be expected to display much more florid and severe cerebrovascular disease. The next question is whether vascular disease may directly cause or 'drive' Alzheimer's disease processes. Many potential mechanisms exist and have been proposed for vascular induction of Alzheimer's disease, including amyloid deposition secondary to ischaemia or peri-infarct inflammation and microglial activation. Abnormal protein glycation may provide a link between diabetes and Alzheimer's disease. Furthermore, there is a growing appreciation of cerebrovascular pathology in Alzheimer's disease such as vascular amyloid deposition and microangiopathy. Apolipoprotein E (ApoE) genotype may also provide a potential explanation for the association. However, although the ApoE $\varepsilon 4$ variant is associated with increased vascular risk, neither lipid levels nor vascular disease have been found to be mediating factors between $\varepsilon 4$ and risk of Alzheimer's disease (Prince et al, 2000).

Although many feasible mechanisms have been proposed for direct links between vascular and Alzheimer's disease processes, pathological evidence so far suggests that they interact principally in their clinical effects. The 'nun study' found no direct association between cerebrovascular disease and level of Alzheimer pathology, but instead found that less Alzheimer pathology was 'required' to produce dementia if cerebrovascular disease was also present (Snowdon et al, 1997). One possible explanation is that memory impairment secondary to hippocampal pathology in Alzheimer's disease is more likely to manifest as clinical dementia if there is also impairment of executive function secondary to vascular subcortical pathology. Executive function impairment is an important, although frequently unmeasured, factor associated with memory decline, and decline in executive function alone, an important potential consequence of cerebrovascular disease, has been suggested as a dementia syndrome in its own right (Royall, 2000).

\section{WHAT CAN WE DO ABOUT IT?}

Considering the length of time vascular disease has been accepted as a common and potentially reversible risk factor for dementia, evidence for interventions to modify this risk has been woefully scant. As summarised in Table 1, the likely success of any intervention depends crucially on the mechanism of association. Of particular interest for clinicians is the extent to which patients with dementia may benefit from treatment of vascular disease or risk factors. However, there has been no optimally designed trial of such interventions. Treatment of vascular risk factors may have an impact on cognitive decline through preventing stroke - and increasing evidence suggests that there may be no upper age limit for stroke prevention (Staessen et al, 2000). In addition, there would be benefit if vascular disease was accelerating the progression of 
Table I Pathways of association between vascular disease and dementia and their implications with respect to preventive or therapeutic interventions

\begin{tabular}{|c|c|c|}
\hline \multirow[t]{2}{*}{ Mechanism of association } & \multicolumn{2}{|c|}{ Potential benefit from modifying vascular risk status } \\
\hline & In those without dementia & In those with dementia \\
\hline Multiple infarcts & Prevention of dementia through stroke prevention & Prevention of further deterioration \\
\hline $\begin{array}{l}\text { Vascular disease accelerating Alzheimer's disease } \\
\text { progression }\end{array}$ & $\begin{array}{l}\text { Prevention/delayed onset of dementia through Alzheimer's } \\
\text { disease deceleration }\end{array}$ & Slowing of further deterioration \\
\hline $\begin{array}{l}\text { Vascular disease accelerating the age of onset of dementia } \\
\text { in the presence of Alzheimer's disease }\end{array}$ & $\begin{array}{l}\text { Prevention/delayed age of onset through reducing risk of } \\
\text { other cognitive impairment }\end{array}$ & Unlikely to have any effect \\
\hline $\begin{array}{l}\text { Common underlying risk factors (e.g. genetic) for vascular } \\
\text { disease and Alzheimer's disease }\end{array}$ & No effect & No effect \\
\hline
\end{tabular}

Alzheimer lesions. On the other hand, if vascular disease predominantly accelerates the age of onset of Alzheimer's disease, treatment should be directed at Alzheimer's disease rather than the vascular component once dementia has developed. This issue has become particularly important with the licensing of pharmacological agents for use in Alzheimer's disease. If stroke continues to be considered as an exclusion criterion, a large number of people with Alzheimer's disease will fail to receive appropriate treatment because of comorbid disease. Whatever lies ahead for the treatment of dementia associated with cerebrovascular disease, the potential for its prevention is likely to be substantial since vascular risk factors have high prevalence rates. A modest reduction in vascular risk across a population could therefore be expected to result in a large reduction in future cases of dementia, if only through delaying its clinical onset. Supporting this, preliminary findings from the large Systolic Hypertension in Europe (Syst-Eur) trial of antihypertensive treatment in older people suggested a $50 \%$ reduction in incident dementia (principally Alzheimer's disease) occurring in those treated with a calcium channel blocker compared with placebo (Forette et al, 1998).

\section{WHERE DO WE GO FROM HERE?}

Further investigation into mechanisms underlying links between vascular and Alzheimer pathological processes is likely to provide important avenues for risk modification. In addition, evidence from adequately designed therapeutic and preventive trials, even if negative, is likely to provide useful information regarding underlying mechanisms. What may well become increasingly apparent is that dementia is a very late stage to be considering useful intervention. One challenge is to develop public health strategies that can affect large populations, reducing vascular risk earlier in life with an aim of decreasing the burden of dementia for that generation
10-20 years later. However, from a clinical standpoint (directed towards individualrather than population-level risk) there will also need to be a move towards identifying cognitive decline at a much earlier stage than dementia. In particular, with regard to vascular disease, the focus on memory decline influenced by Alzheimer's disease will need to shift towards an appreciation of executive function impairment (Bowler \& Hachinski, 2000).

What, therefore, is vascular dementia? It is certainly a convenient subject heading for a rapidly accelerating field of research. What is less clear is whether it retains any usefulness as a diagnosis. Vascular dementia implies a primary cause (cerebrovascular disease) linked with a specific consequence (a distinguishable dementia syndrome). However, increasing evidence suggests that dementia associated with vascular disease involves a very broad spectrum of manifestations, from strategic infarct syndromes to Alzheimer's disease. In addition, although vascular disease is a powerful risk factor for dementia, it is questionable how

Table 2 A life-course model for the aetiology of cognitive decline and dementia

\begin{tabular}{|c|c|c|c|c|c|}
\hline \multirow[t]{2}{*}{ Genetic factors } & \multirow[t]{2}{*}{ Early-life factors } & \multirow[t]{2}{*}{ Mid-life factors } & \multicolumn{3}{|c|}{ Later-life factors } \\
\hline & & & Physiology & Pathology & Environment \\
\hline ApoE & In utero environment & Blood pressure & Cerebral perfusion & Large vessel disease & Comorbid disease \\
\hline \multirow[t]{7}{*}{ Other factors } & Childhood environment & Lipid levels & Inflammation & Small vessel disease & Depression \\
\hline & Education & Insulin resistance & Neuroendocrine environment & Infarction & Social support \\
\hline & Socio-economic status & Glucose intolerance & Oxidative stress & Ischaemia & Access to health care \\
\hline & & Lifestyle factors (smoking, & Excitotoxicity & Amyloid deposition & Cultural factors \\
\hline & & exercise, etc.) & & Tau phosphorylation & Socio-economic status \\
\hline & & Socio-economic status & & & \\
\hline & \multicolumn{2}{|c|}{ Developing 'premorbid' cognitive function } & \multicolumn{3}{|c|}{ Early cognitive decline $\rightarrow$ Detectable cognitive decline $\rightarrow$ Dementia } \\
\hline
\end{tabular}

ApoE, apolipoprotein E. 
often it is actually the sole cause of dementia in the absence of underlying vulnerability or comorbid disease. Dementia research has suffered, in common with other areas of epidemiology, from a simplistic conceptual framework of risk factor-outcome relationships. What is increasingly evident is that there are many potential factors operating across an entire life course (and possibly extending back into previous generations) which may influence or mediate each other's effects (Table 2). Designing appropriate studies and systems of analysis to take into account these multiple influences and interactions is likely to provide a major challenge for future research.

In the meantime, what is to be done with vascular dementia? A recent editorial has suggested that, rather than tinkering with existing criteria, they should be discarded and replaced wholesale (Bowler \& Hachinski, 2000). Certain syndromes can be reasonably considered as diagnoses, for example dementia due to strategic infarcts or genetic disorders such as CADASIL (cerebral autosomal dominant arteriopathy with subcortical infarcts and leucoencephalopathy). However, for the majority of vascular dementia syndromes arising in later life, although these may manifest as 'pure' disorders, it is questionable whether valid distinctions can truly be drawn between sub-categories or between vascular dementia and Alzheimer's disease. The result is that clinical research diagnostic criteria for vascular dementia involve a high degree of subjective judgement (Skoog \& Aevarsson, 2000), leading inevitably to poor agreement between raters (Lopez et al, 1994) and between different diagnostic instruments (Chui et al, 2000). For depression, we do not feel the need to define 'life event depression' or 'poor physical health depression'; rather, we direct treatment at the symptoms and, holding in mind our formulation of possible underlying causes, attempt to modify potential maintaining factors and risk factors for recurrence. Perhaps there may be lessons to be learned for cognitive impairment and dementia. A complex picture of overlapping and interacting pathological entities is one of the challenges and rewards of health care for older age groups that we enthuse about to students and trainees. Is a diagnostic system that attempts to separate these processes into artificially pure diagnoses doing us or them - or those we treat - any favours?

\section{CLINICAL IMPLICATIONS}

- Modification of vascular risk status in mid-life is likely to be an important means of reducing the subsequent risk of dementia for a population but evidence is lacking for an effect on the course of dementia, once this has developed.

- The usefulness of vascular dementia as a diagnostic category is questionable since it involves a high degree of subjective judgement, subsumes a large number of potentially heterogeneous conditions and overlaps considerably with Alzheimer's disease.

- The current system of classifying dementia into mutually exclusive subtypes poorly reflects mixed disease in older age groups and has become an important issue since these 'diagnoses' now determine eligibility for pharmacological intervention.

\section{LIMITATIONS}

- The review of the literature is selective and limited.

- This review looks at the relationship between vascular disease and dementia at a population level rather than at specific discrete syndromes within vascular dementia.

- The focus of this review is on the relationship between cerebrovascular disease and Alzheimer's disease as two overlapping disorders and does not take into account other comorbid pathology.

ROBERT STEWART, MRCPsych, Institute of Psychiatry, De Crespigny Park, Denmark Hill, London SE5 8AF, UK. Tel: +44 (0)20 7848 0240; fax: +44 (0)20 770I 0167; e-mail: r.stewart@iop.kcl.ac.uk

(First received I5 March 2000, final revision 26 April 200I, accepted 27 April 200I)

\section{REFERENCES}

Bowler, J.v. \& Hachinski, v. (2000) Criteria for vascular dementia: replacing dogma with data. Archives of Neurology, 57, 170-17|.

Brun, A. (2000) The neuropathology of vascular dementia. In Cerebrovascular Disease and Dementia: Pathology, Neuropsychiatry and Management (eds E. Chiu, L. Gustafson, D. Ames, et al), pp. 69-76. London: Martin Dunitz.

Chui, H. C., Mack, W., Jackson, J. E., et al (2000) Clinical criteria for the diagnosis of vascular dementia. Archives of Neurology, 57, 191-196.

Elias, M. F., Wolf, P. A., D’Agostino, R. B., et al (1993) Untreated blood pressure level is inversely related to cognitive functioning: the Framingham study. American Journal of Epidemiology, I38, 353-364.

Forette, F., Seux, M.-L., Staessen, J. A., et al (1998) Prevention of dementia in randomised double-blind placebo-controlled Systolic Hypertension in Europe (Syst-Eur) trial. Lancet, 352, I347-1351.

Hulette, C., Nochlin, D., McKeel, D., et al (1997) Clinical-neuropathological findings in multi-infarct dementia: a report of six autopsied cases. Neurology, $\mathbf{4 8}$, 668-672.

Kokmen, E., Whistman, J. P., O'Fallon, W. M., et al (1996) Dementia after ischemic stroke: a population- based study in Rochester, Minnesota (1960-1984). Neurology, 19, 154-159.

Konno, S., Meyer, J. S., Terayama, Y., et al (1997) Classification, diagnosis and treatment of vascular dementia. Drugs and Aging, II, 36I-373.

Lopez, O. L., Larumbe, M. R., Becker, J.T., et al (1994) Reliability of NINDS-AIREN clinical criteria for the diagnosis of vascular dementia. Neurology, 44, 1240-1245.

McKhann, G., Drachman, D., Folstein, M., et al (1984) Clinical diagnosis of Alzheimer's disease. Report of the NINCDS-ADRDA Work group under the auspices of the Department of Health and Human Services Task Force on Alzheimer's Disease. Neurology, 34, 939-944.

Notkola, L-M., Sulkava, R., Pekkanen, J., et al (1998) Serum total cholesterol, apolipoprotein E 4 allele, and Alzheimer's disease. Neuroepidemiology, 17, 14-20.

Ott, A., Slooter, A. J. C., Hofman, A., et al (1998) Smoking and the risk of dementia and Alzheimer's disease in a population-based cohort study: the Rotterdam Study. Lancet, 35I, 1840-1843.

Pohjasvaara, T., Mäntylä, R., Aronen, H. J., et al (1999) Clinical and radiological determinants of prestroke cognitive decline in a stroke cohort. 
Journal of Neurology, Neurosurgery and Psychiatry, 67, 742-748.

Prince, M., Lovestone, S., Cervilla, J., et al (2000) The association between APOE and dementia is mediated neither by vascular disease nor its risk factors in an aged cohort of survivors with hypertension. Neurology, 54, 397-402.

Royall, D. R. (2000) Executive cognitive impairment: a novel perspective on dementia. Neuroepidemiology, $\mathbf{1 9}$, 293-299.
Skoog, I. \& Aevarsson, O. (2000) Epidemiology of vascular dementia in Europe. In Cerebrovascular Disease and Dementia: Pathology, Neuropsychiatry and

Management (eds E. Chiu, L. Gustafson, D. Ames, et al), pp. 15-24. London: Martin Dunitz.

_ , Lernfelt, B., Landahl, S., et al (1996) 15-year longitudinal study of blood pressure and dementia. Lancet, 347, ||4|-||45.
Snowdon, D. A., Greiner, L. H., Mortimer, J. A., et al (1997) Brain infarction and the clinical expression of Alzheimer disease. JAMA, 277, 813-817.

Staessen, J. A., Gasowski, J., Wang, J. G., et al (2000) Risks of untreated and treated isolated systolic hypertension in the elderly: meta-analysis of outcome trials. Lancet, 355, 865-872.

Stewart, R. (1998) Cardiovascular factors in Alzheimer's disease. Journal of Neurology, Neurosurgery and Psychiatry, 65, 143-147. 